Service social

\title{
Le coffre à outils du chercheur débutant : Guide d'initiation au travail intellectuel, par Jocelyn Létourneau, Toronto, Oxford University Press, 1989, 227 pages.
}

\section{Suzanne Bérubé}

Volume 38, numéro 2-3, 1989

Les politiques sociales

URI : https://id.erudit.org/iderudit/706445ar

DOI : https://doi.org/10.7202/706445ar

Aller au sommaire du numéro

Éditeur(s)

École de service social de l'Université Laval

ISSN

1708-1734 (numérique)

Découvrir la revue

Citer ce compte rendu

Bérubé, S. (1989). Compte rendu de [Le coffre à outils du chercheur débutant : Guide d'initiation au travail intellectuel, par Jocelyn Létourneau, Toronto, Oxford University Press, 1989, 227 pages.] Service social, 38(2-3), 317-319. https://doi.org/10.7202/706445ar d'utilisation que vous pouvez consulter en ligne.

https://apropos.erudit.org/fr/usagers/politique-dutilisation/ 


\section{RECENSIONS}

Le coffre à outils du chercheur débutant: Guide d'initiation au travail intellectuel, par Jocelyn LÉTOURNEAU, Toronto, Oxford University Press, 1989, 227 pages.

L'auteur n'aurait pu intituler son ouvrage de façon plus appropriée. Ce livre se veut un guide pratique d'initiation à diverses techniques et méthodes dans le domaine de la recherche et du travail intellectuel. II s'adresse particulièrement à des étudiants de niveau collégial ou universitaire devant répondre à diverses exigences académiques, et aux novices dans le domaine de la recherche et du travail intellectuel méthodique.

L'auteur est professeur en histoire canadienne et en méthodologie de I'histoire à l'Université Laval. Constatant chez ses étudiants le besoin d'ouvrages de référence, il a mis au point ce guide qui se veut pratique et utile, adapté aux tendances actuelles de la recherche, visant des objectifs d'apprentissage élevés, et utilisable par des étudiants provenant de différentes disciplines, bien qu'il nous semble plus approprié pour les étudiants des sciences sociales et humaines.

La structure de l'ouvrage est limpide et pratique. II est composé de deux grandes sections qui se divisent en quatorze chapitres autonomes pouvant être consultés séparément.

Dans la première section, sont rassemblés les "savoir-faire pratiques". Ce sont des techniques de travail, des méthodes, des conseils et des trucs opérationnels concernant neuf activités particulières. L'auteur vise à initier le lecteur à une démarche méthodique, il tente de fournir des indications de base et non de renseigner le spécialiste. Il nous y apprend comment faire la recension d'une lecture, l'analyse d'un document écrit, d'un tableau statistique, ou d'un document autobiographique. Il expose les rudiments de l'enquête auprès d'informateurs. L'analyse du document iconographique, de l'objet matériel et de la carte ancienne y est aussi présentée. Un important chapitre sur la recherche documentaire conventionnelle ou informatisée constitue, à mon avis, le cœur de cette partie.

La deuxième section de l'ouvrage comprend cinq chapitres dans lesquels l'auteur expose les grandes étapes de la réalisation d'un rapport de recherche ou d'un travail long. II traite également de la manière de circonscrire un sujet de recherche, des composantes d'une stratégie de recherche, du plan de travail et de l'art de communiquer sa pensée par écrit. Outre ces deux grandes sections, le volume comporte une présentation claire de son objet et des conseils à I'utilisateur. Il est agrémenté d'une table des matières détaillée et utile. Quelques chapitres seulement comportent une bibliographie com- 
plémentaire, permettant au lecteur de poursuivre son cheminement. La généralisation de cette formule aurait été fort pertinente, vu la philosophie de ce document, et aurait favorisé l'élargissement du public intéressé. Deux annexes complètent ce guide, elles portent sur la présentation des références bibliographiques et des notes en bas de page.

Adoptant parfois un ton professoral, ce volume est écrit dans un style sobre et clair. Les objectifs poursuivis par l'auteur et sa perspective didactique caractérisent son raisonnement et sa démarche. Cette dernière est essentiellement informative et descriptive. Pour chaque activité ou techniquèè à laquelle il nous initie, l'auteur en définit d'abord la nature, les objectifs, les caractéristiques et exigences. II décompose ensuite le processus en séquences logiques et progressives et il en explique, interprète et détaille chacune des étapes de réalisation. II accompagne le tout de textes explicatifs et de tableaux synthèse ou récapitulatifs qui ajoutent d'ailleurs considérablement à la compréhension et à la vision d'ensemble de l'activité analysée. Il illustre le résultat final, mettant en pratique les méthodes enseignées. Les exemples sont utilisés de façon très pertinente et d'intérêt assez général, puisque tirés d'une littérature relative à l'histoire. Finalement, une documentation importante sous-tend chaque thème; le choix de l'auteur de s'entourer de onze collaborateurs, chacun étant spécialiste dans son domaine spécifique, contribue vraisemblablement à la densité et à la richesse de l'ouvrage.

Quelques chapitres ont retenu plus particulièrement mon attention, dont celui portant sur la manière de se documenter et de maximiser son travail en bibliothèque. Posant d'abord le problème de la quantité des publications et de la dispersion de la documentation, l'auteur nous propose une démarche méthodique en six étapes. II passe ensuite en revue les caractéristiques des diverses sources documentaires les plus courantes (répertoires, index, bibliographies, recherche informatisée, etc.) et nous livre seize trucs pour maximiser le travail en bibliothèque. Un véritable coffre à outils documentaires des ouvrages les plus fréquemment utilisés par les chercheurs en sciences humaines et sociales termine ce chapitre. Fort exhaustif et, à ma connaissance, unique en son genre, il s'agit là d'un outil précieux tant pour le débutant que pour le chercheur chevronné qui désire s'assurer de la systématisation de sa démarche de recherche d'informations.

De même, la deuxième partie de l'ouvrage m'a particulièrement intéressée. L'auteur nous y présente de façon claire, complète et détaillée toutes les étapes de la réalisation d'un travail de recherche. Pour chacune, il expose les objectifs, les tâches inhérentes, les questions concrètes auxquelles le chercheur doit répondre. La démarche est disséquée, démontrée, justifiée et, par conséquent, sa compréhension simplifiée. L'auteur met l'accent sur la nécessité d'un sujet précis et opérationnel, il met en lumière l'importance de la position de problème et de la stratégie de recherche, et démontre la nature essentielle du plan de travail, ses étapes d'élaboration et son utilité. II termine en nous rappelant qu'avoir des idées brillantes ne suffit pas, il faut aussi maîtriser l'art de communiquer sa pensée par écrit. Il indique des principes, suggère des règles, propose des trucs afin de guider le rédacteur soucieux de valoriser ses idées et arguments et de capter l'attention du lecteur. 
Létourneau est passé maître dans cet art de convaincre et de séduire, tout comme dans celui de la pédagogie. La singularité de cet ouvrage réside dans I'habileté de l'auteur à décortiquer le processus d'une technique ou d'une activité intellectuelle. Fort rigoureux dans sa démarche, il arrive à transmettre de façon simple, claire et précise, une matière qui pourrait être qualifiée d'aride et qui a fait l'objet de peu de publications. La démarche de l'auteur et la structure de son ouvrage suivent une logique sans faille; l'œuvre ne pourrait être plus systématique.

En définitive, il s'agit d'un excellent ouvrage de consultation qui regorge d'outils pratiques. Véritable exemple de travail méthodique, il est accessible au public auquel il s'adresse et même à l'autodidacte, ou encore à ceux et celles qui ne se sont pas astreints depuis un certain temps aux règles des travaux académiques.

Suzanne BÉRUBÉ

Étudiante à la maîtrise,

École de service social, Université Laval.

Protection de l'enfance - Réalité de l'intervention, par Robert Dubé et Marjolaine SAINT-JULES, Montréal, Gaëtan Morin Éditeur, Fondation des enfants maltraités du Québec inc., 1987, 242 pages.

Ce livre a pour objet la problématique des enfants maltraités et les systèmes de protection en place pour remédier aux insuffisances de leur milieu familial. Les trois sections du volume sont agencées de façon à faire cheminer le lecteur à travers une prise de conscience du problème, un regard sur les aspects dynamiques de l'intervention en protection de l'enfance et une vue d'ensemble des mesures de protection actuellement en place au Québec. Nous constatons une grande rigueur dans la démarche des auteurs, démarche basée sur une revue approfondie des écrits et une information très complète sur le sujet.

La première partie du volume intitulée "le problème ", présente la problématique sous différents angles. Ainsi, l'observation de l'enfant à travers I'histoire nous permet de saisir la considération qu'ont eue les humains à l'endroit de l'enfant et la place qui lui a été réservée au cours des siècles.

La définition et la recherche des causes de la maltraitance procurent au lecteur une occasion de bien circonscrire les abus physiques, la négligence et les abus sexuels. On y expose l'origine de ces problématiques et les facteurs explicatifs sous-jacents. Quant à la section portant sur les conséquences de la maltraitance, le lecteur ne pourra demeurer indifférent devant une telle présentation des séquelles et limites qui marqueront l'enfant victime, et ce, tout au cours de son développement. 